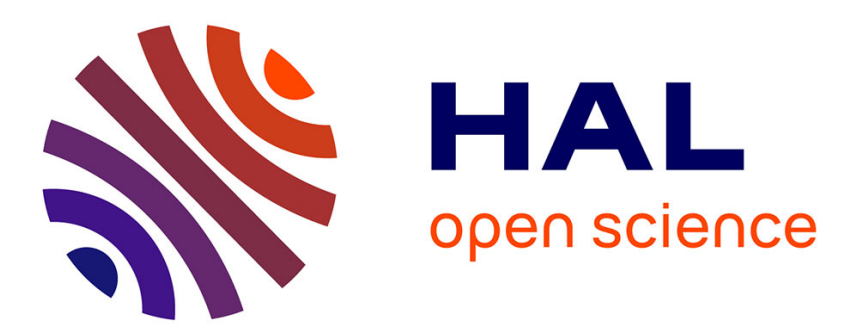

\title{
Les paragenèses de smectites et de zéolites dans une série pyroclastique d'âge éocène moyen de l'Océan Indien (D.S.P., leg. 26, site 253)
}

Dominique Besse, Alain Desprairies, Célestine Jehanno, Venkatarathman Kolla

\section{To cite this version:}

Dominique Besse, Alain Desprairies, Célestine Jehanno, Venkatarathman Kolla. Les paragenèses de smectites et de zéolites dans une série pyroclastique d'âge éocène moyen de l'Océan Indien (D.S.P., leg. 26, site 253). Bulletin de Mineralogie, 1981, 104 (1), pp.56 - 63. 10.3406/bulmi.1981.7544 . hal-03413037

\section{HAL Id: hal-03413037 \\ https://hal.science/hal-03413037}

Submitted on 3 Nov 2021

HAL is a multi-disciplinary open access archive for the deposit and dissemination of scientific research documents, whether they are published or not. The documents may come from teaching and research institutions in France or abroad, or from public or private research centers.
L'archive ouverte pluridisciplinaire HAL, est destinée au dépôt et à la diffusion de documents scientifiques de niveau recherche, publiés ou non, émanant des établissements d'enseignement et de recherche français ou étrangers, des laboratoires publics ou privés. 


\section{Les paragenèses de smectites et de zéolites dans une série} pyroclastique d'âge éocène moyen de l'Océan Indien (D.S.P., leg. 26, site 253)

Dominique Besse, Alain Desprairies, Célestine Jehanno, Venkatarathman Kolla

\section{Citer ce document / Cite this document :}

Besse Dominique, Desprairies Alain, Jehanno Célestine, Kolla Venkatarathman. Les paragenèses de smectites et de zéolites dans une série pyroclastique d'âge éocène moyen de l'Océan Indien (D.S.P., leg. 26, site 253). In: Bulletin de Minéralogie, volume 104, 1, 1981. pp. 56-63;

doi : https://doi.org/10.3406/bulmi.1981.7544

https://www.persee.fr/doc/bulmi_0180-9210_1981_num_104_1_7544

Fichier pdf généré le 27/11/2019 


\begin{abstract}
Zeolites and clay minerals formed by submarine alteration of a thick hyaloclastic basaltic sequence have been studied by X-ray diffraction and by Scanning Electron Microscope with X-ray dispersive energy attachment. Zeolite compositions show three successive zones from the top to the base of the sequence : phillipsite zone, clinoptilolite-mordenite zone and analcime-clinoptilolite zone. Among the clay minerals, smectites are dominant : well crystallized, they are Fe-rich near the top and Mg-rich near the base of the sequence. Locally, celadonite is present. This zonation of authigenic mineral assemblages can be assigned to hydrothermal alteration in a high temperature geothermal area (approximately $200^{\circ} \mathrm{C} / \mathrm{km}$ ).
\end{abstract}

\title{
Résumé
}

Les zéolites et les minéraux argileux, provenant d'une altération sous-marine d'une épaisse série de hyaloclastites basaltiques, ont été étudiés au microscope électronique à balayage couplé à un spectromètre à dispersion d'énergie et par diffraction des rayons X. Les analyses montrent une évolution de la nature des zéolites selon la profondeur. Trois zones se succèdent de haut en bas dans la séquence : une zone à phillipsite, une zone à clinoptilolite-mordenite, et une zone à analcimeclinoptilolite. Parmi les minéraux argileux, les smectites sont prédominantes ; bien cristallisées, elles sont ferrifères au sommet et magnésiennes à la base de la série. On note, localement, la présence de céladonite. Cette zonation dans les paragenèses observées semble pouvoir être reliée à une altération hydrothermale dans une aire géothermique de relative haute température (environ $200^{\circ} \mathrm{C} / \mathrm{km}$ ). 


\title{
Les paragenèses de smectites et de zéolites dans une série pyroclastique d'âge éocène moyen de l'Océan Indien (D.S.D.P., Leg 26, Site 253) (*)
}

\author{
par Dominique BESSE $\left({ }^{1}\right)$, Alain DESPRAIRIES $\left({ }^{1}\right)$, Célestine JEHANNO $\left({ }^{2}\right)$ \\ et Venkatarathman KOLla $\left({ }^{3}\right)$, \\ (1) E.R.A. 765 du C.N.R.S., Physico-chimie des processus bio-sédimentaires, \\ Laboratoire de Géochimie des roches sédimentaires, \\ Université de Paris-Sud, 91405 Orsay, France. \\ ${ }^{2}$ ) Centre des faibles radioactivités. \\ Laboratoire C.E.A./C.N.R.S., 91190 Gif-sur-Yvette, France. \\ ${ }^{3}$ ) Lamont Doherty Geological Observatory, \\ Columbia University, Palisades, New York, U.S.A.
}

\begin{abstract}
Résumé. - Les zéolites et les minéraux argileux, provenant d'une altération sous-marine d'une épaisse série de hyaloclastites basaltiques, ont été étudiés au microscope électronique à balayage couplé à un spectromètre à dispersion d'énergie et par diffraction des rayons X. Les analyses montrent une évolution de la nature des zéolites selon la profondeur. Trois zones se succèdent de haut en bas dans la séquence : une zone à phillipsite, une zone à clinoptilolite-mordenite, et une zone à analcime-clinoptilolite. Parmi les minéraux argileux, les smectites sont prédominantes; bien cristallisées, elles sont ferrifères au sommet et magnésiennes à la base de la série. On note, localement, la présence de céladonite. Cette zonation dans les paragenèses observées semble pouvoir être reliée à une altération hydrothermale dans une aire géothermique de relative haute température (environ $200^{\circ} \mathrm{C} / \mathrm{km}$ ).
\end{abstract}

Mots clés : zéolites, smectites, hyaloclastites, hydrothermalisme, Océan Indien.

Smectite-zeolite associations in a middle Eocene hyaloclastic sequence from the Indian Ocean (Leg 26, site 253).

Abstract. - Zeolites and clay minerals formed by submarine alteration of a thick hyaloclastic basaltic sequence have been studied by X-ray diffraction and by Scanning Electron Microscope with X-ray dispersive energy attachment. Zeolite compositions show three successive zones from the top to the base of the sequence : phillipsite zone, clinoptilolite-mordenite zone and analcime-clinoptilolite zone. Among the clay minerals, smectites are dominant : well crystallized, they are Fe-rich near the top and Mg-rich near the base of the sequence. Locally, celadonite is present. This zonation of authigenic mineral assemblages can be assigned to hydrothermal alteration in a high temperature geothermal area (approximatly $200^{\circ} \mathrm{C} / \mathrm{km}$ ).

Key words : zeolites, smectites, hyaloclastites, hydrothermal, Indian Ocean.

\section{INTRODUCTION}

Le site 253 du leg 26 (Figure 1) se situe sur la Ninety east Ridge (Lat $24^{\circ} 52,65^{\prime} \mathrm{S}$, long. $87^{\circ} 21,97^{\prime} \mathrm{E}$ ). Au cours du forage, effectué sous 1962 mètres d'eau, 558 mètres de sédiments ont été traversés. Les 153 premiers mètres sont constitués par une zone carbonatée à nannoplancton et Foraminifères, d'âge quaternaire à éocène moyen; ils surmontent 405 mètres d'une série de hyaloclastites de l'Éocène moyen.

\section{Données pétrographiques.}

La puissante série de hyaloclastites qui sert de cadre à notre étude, a été décrite avec précision par McKelvey et Fleet (1974). Ces auteurs l'ont subdivisée, de

(*) Communication présentée le 13 mars 1980 à la réunion de la S.F.M.C. qui avait pour thème "Minéralogie sédimentaire : néoformation, diagenèse, marqueurs du paléoenvironnement ».

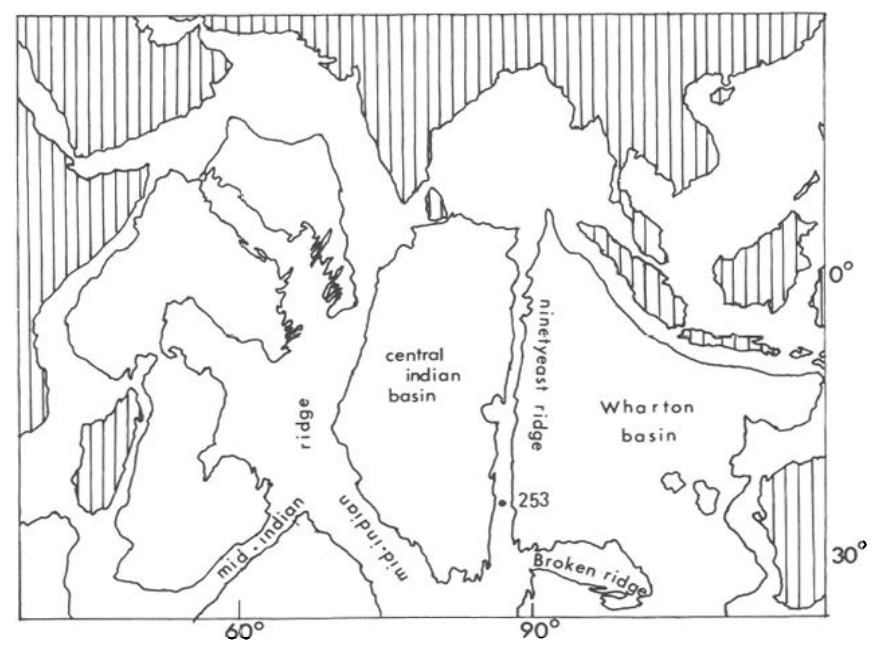

FIG. 1. - Localisation du site étudié.

haut en bas, en 6 unités lithologiques, aux limites quelquefois imprécises (Figure 2). Dans les unités supérieures, bien stratifiées, l'épaisseur des bancs varie de 


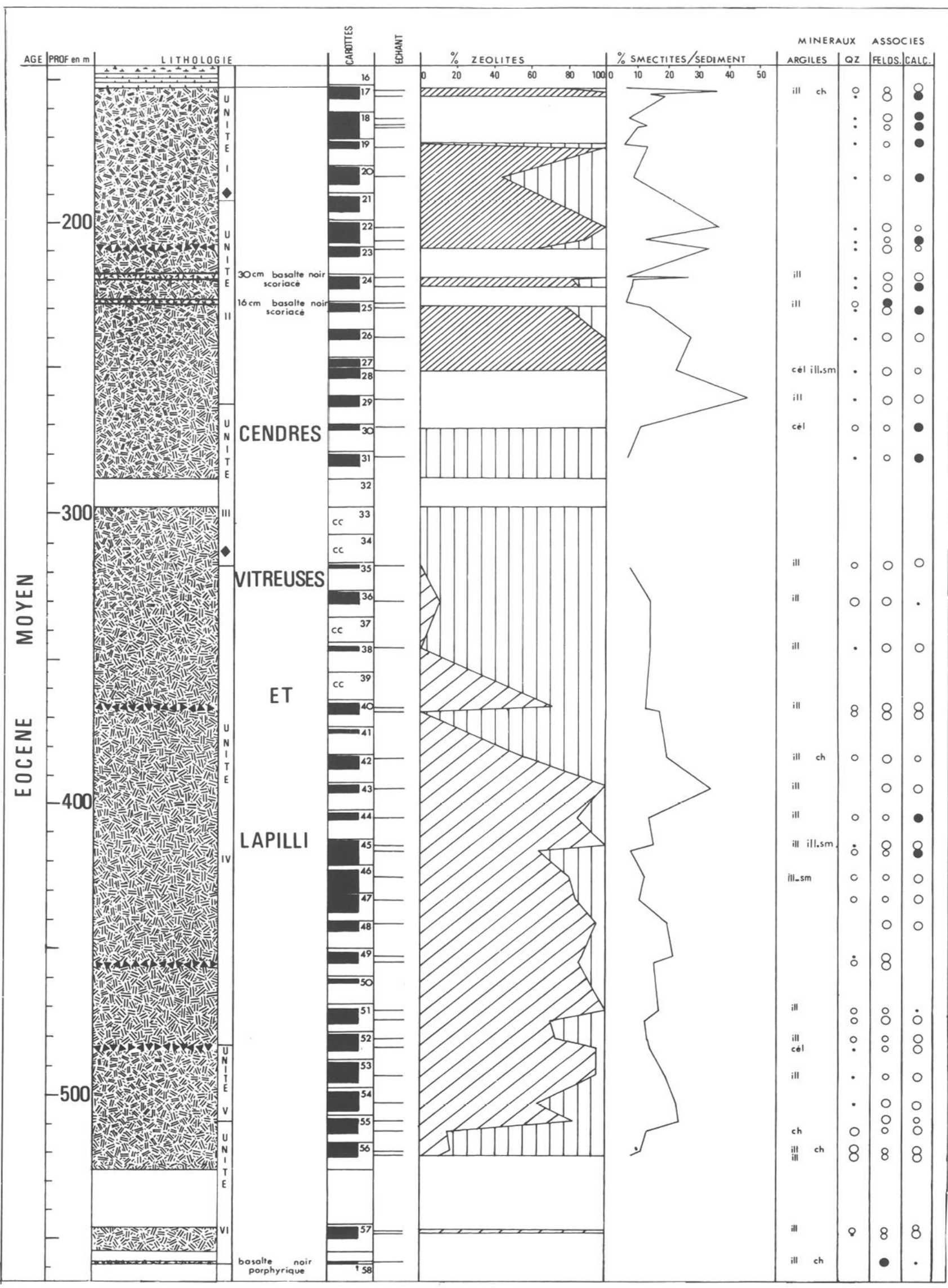

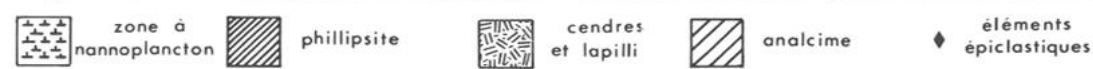

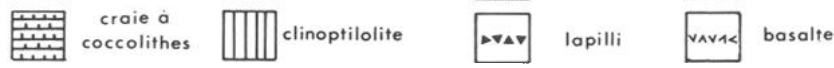

ill. illite

- $0-5 \%$

ch. chlorite

- $50-100 \%$

FIG. 2. - Lithologie et minéralogie de la série des hyaloclastites. 
$2,5 \mathrm{~m}$ à $1 \mathrm{~cm}$. Dans les unités inférieures, la stratigraphie est moins visible et beaucoup de passages de bancs sont graduels. Les structures sédimentaires sont rares dans toute la séquence. Les unités I (153,30 à $192,30 \mathrm{~m})$ et III $(262,60$ à $318 \mathrm{~m})$ sont formées d'éléments pyroclastiques, mais aussi épiclastiques, alors que les unités II (192,30 à 262,60 m), IV (318 à $423 \mathrm{~m}$ ), V $(483$ à $509 \mathrm{~m})$ et VI $(509$ à $558 \mathrm{~m})$ sont purement pyroclastiques. La séquence se termine à $558 \mathrm{~m}$ de profondeur par un basalte noir, porphyrique, fortement altéré ( $46 \pm 3 \mathrm{M}$.A./BP). On note également, interstratifiées dans l'unité II, 2 fines venues d'un basalte noir scoriacé, relativement frais.

\section{Données paléontologiques.}

La faune est abondante dans toute la série, mais irrégulièrement répartie. Les microfossiles (y compris les nannofossiles) sont présents dans toutes les unités excepté dans les lapilli des unités II et IV. Les macrofossilcs sont très nombreux et bien préservés dans les unités II et III; ils sont absents de l'unité V et on les retrouve en débris dans les autres unités (I, IV et VI).

\section{Milieu de sédimentation.}

Les nannofossiles et Foraminifères correspondent à un environnement subtropical et la présence abondante de «Discocyclina» dans toutes les unités indique une profondeur faible, de l'ordre de 150 mètres (Davies et al., 1974). Le taux de sédimentation est élevé $(\simeq 100 \mathrm{~m} /$ M.A.) et pour McKelvey et al. (1974), le mécanisme de formation des hyaloclastites est tout à fait comparable à celui décrit par Tazieff (1972) lors des éruptions sous-marines à faible profondeur, autour de l'île de Surtsey.

\section{MÉTHODE D'ÉTUDES}

52 échantillons répartis dans toute la série ont été analysés par diffraction des rayons $\mathrm{X}$ (radiation cobalt), et à l'aide d'un microscope électronique à balayage (CAMECA) couplé à un spectromètre à dispersion d'énergie (E.D.S.).

En diffractrométrie $\mathrm{X}$, les études de chaque échantillon ont porté systématiquement sur la poudre totale, sur les poudres décarbonatées de fraction inférieure à 35 et $2 \mu \mathrm{m}$ et sur des agrégats orientés de la fraction argileuse.

Dans les diffractogrammes de poudre totale, nous avons estimé les pourcentages des différentes zéolites par rapport au sédiment total, mais aussi entre elles, par les hauteurs relatives, ou les surfaces relatives, de pics caractéristiques de ces minéraux. Ces deux procédés de mesure semi-quantitative sont bien sûr approximatifs, mais donnent des résultats comparables et suffisamment reproductibles. Nous avons évalué de la même manière (Figure 2) la proportion des argiles (en choisissant la raie de structure à $4,55 \AA$ ) et la teneur des autres phases minérales (quartz, feldspaths, carbonates).

Dans les diffractogrammes de poudre décarbonatée, les paramètres $b$ moyens, donnés par les bandes 060 de certains minéraux argileux, ont été reliés aux teneurs en fer et magnésium de leurs couches octaédriques (Desprairies, à paraître).

Enfin, sur les agrégats orientés, après différents traitements classiques (glycérine, éthylène-glycol, hydrazine et cuisson), nous avons distingué et estimé, comme ci-dessus, les espèces argileuses, minéraux simples ou interstratifiés.

En microscopie électronique à balayage et en analyse spectrométrique, seuls les échantillons les plus intéressants, soit une vingtaine, ont été étudiés sous 3 formes : en fragments (quelques dizaines de $\mathrm{mm}$ ), en grains nettoyés aux ultra-sons et triés sous la loupe binoculaire selon un critère de couleur ou morphologique, en poudre ultra-fine séparée par centrifugation et ne renfermant plus, d'après le contrôle diffractométrique, que la fraction argileuse du sédiment.

Les échantillons ont été métallisés au carbone et parfois à l'or. Sur les fragments et les grains triés, nous avons obtenu les compositions chimiques d'amas plus ou moins individualisés de minéraux argileux et des zéolites. En pastillant les poudres, nous avons évalué la composition chimique moyenne des minéraux argileux de chaque échantillon.

Tous les résultats fournis par l'analyse spectrométrique sont semi-quantitatifs. L'erreur relative est de $5 \%$ pour les éléments de teneur en poids $\geqslant$ à $10 \%$; de $10 \%$ pour les éléments de teneur comprise entre 10 et $5 \%$, et enfin de $20 \%$ pour les éléments mineurs $(\leqslant 5 \%)$. Ces résultats ont tous été normalisés à $100 \%$ d'oxydes, c'est-à-dire sans tenir compte des ions hydroxyles ni de l'eau résiduelle.

\section{MINERALOGIE DES COMPOSANTS DES DIFFÉRENTES UNITÉS}

\section{A) Les produits néoformés essentiels.}

\section{1) Les zéolites (Figures 2 et 3; tableau I).}

On les rencontre en remplissage des vacuoles et des fissures de la roche ou en ciment des fragments vitreux. Nous avons mis en évidence 3 associations de zéolites.

\begin{tabular}{|c|c|c|c|c|c|c|}
\hline Echantillons & $: \mathrm{SiO}_{2}$ & $\mathrm{Al}_{2} \mathrm{O}_{3}$ & $\mathrm{Na}_{2} \mathrm{O}$ & $\mathrm{K}_{2} \mathrm{O}$ & $\mathrm{CaO}$ & $\mathrm{FeO}$ \\
\hline $\begin{array}{l}\text { Phillipsite } \\
\mathrm{n}^{\circ}{ }_{23-1}\end{array}$ & 67.40 & 19.45 & 7.46 & 5.70 & - & - \\
\hline $\begin{array}{c}\text { Mordenite } \\
\mathrm{n}^{\circ} 42-1\end{array}$ & 75.35 & 14.11 & 6.65 & - & 2.55 & 1.34 \\
\hline $\begin{array}{l}\text { Clinoptilolite } \\
n^{\circ} 30-1\end{array}$ & 74.60 & 15.49 & 5.15 & 1.37 & 1.81 & 1.58 \\
\hline $\begin{array}{l}\text { Clinoptilolite } \\
\text { Calcique } \\
\text { n } 49-2\end{array}$ & 78.52 & 14.80 & 2.59 & - & 4.10 & - \\
\hline $\begin{array}{c}\text { Analcime } \\
n^{\circ} 53-4\end{array}$ & 65.88 & 22.06 & 12.06 & - & - & - \\
\hline
\end{tabular}

TABleau I. - Exemples de composition chimique de zéolites (analyses spectrométriques). 


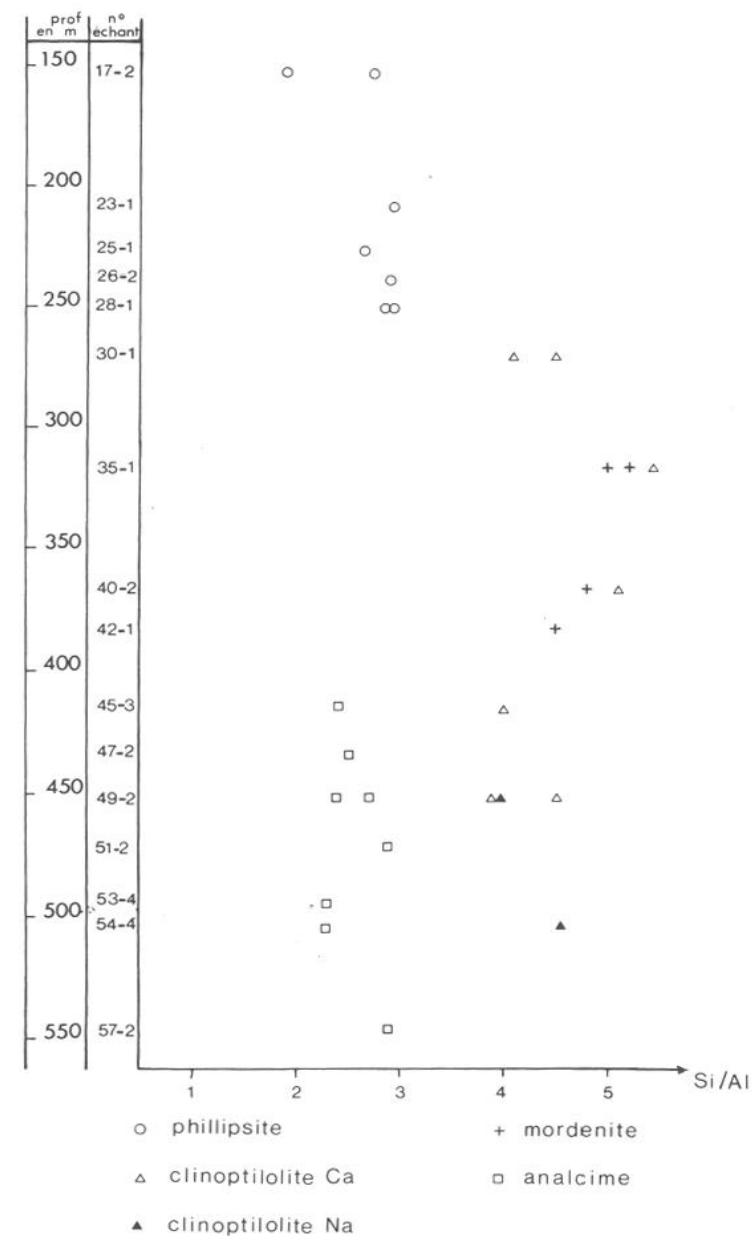

FIG. 3. - Rapport Si/Al des zéolites en fonction de la profondeur (d'après les formules structurales calculées à partir des analyses spectrométriques).

a) Unités basales. (VI, V et la base de IV). On trouve à la fois une zéolite sodique, l'analcime, et une zéolite très siliceuse (fortement calcique), la clinoptilolite. Ces deux zéolites représentent $25 \%$ du sédiment total et l'analcime compose à elle seule près de $80 \%$ de l'association.

- L'analcime a une texture massive et au microscope électronique à balayage, elle se présente souvent en gros cristaux $(20-30 \mu \mathrm{m})$ accolés (Figure $4 a$ ) avec quelques faces bien développées de formes dérivées du cube. Nous n'avons pas retrouvé la texture fibro-radiée de l'analcime décrite par McKelvey et al. (1974), dans le haut de l'unité IV. Nous pensons qu'il s'agit d'une confusion par ces auteurs avec la mordenite, bien présente, elle, dans les unités moyennes.

D'après la formule structurale, calculée sur 96 oxygènes à partir des analyses chimiques, le rapport $\mathrm{Si} / \mathrm{Al}$, varie entre 2,3 et $2,9(50 \%$ de 28 analyses entre 2,5 et 2,6). Par la méthode de Saha (1959), fondée sur la diffraction des rayons $X$, nous avons trouvé un rapport compris entre 2,10 et 2,80 et un maximum d'analyses situé au-delà de 2,40 .

- La clinoptilolite se présente en cristaux tabulaires bien individualisés, de petite taille. Son rapport $\mathrm{Si} / \mathrm{Al}$ est compris entre 4 et 5,40 et elle est fortement sodique.
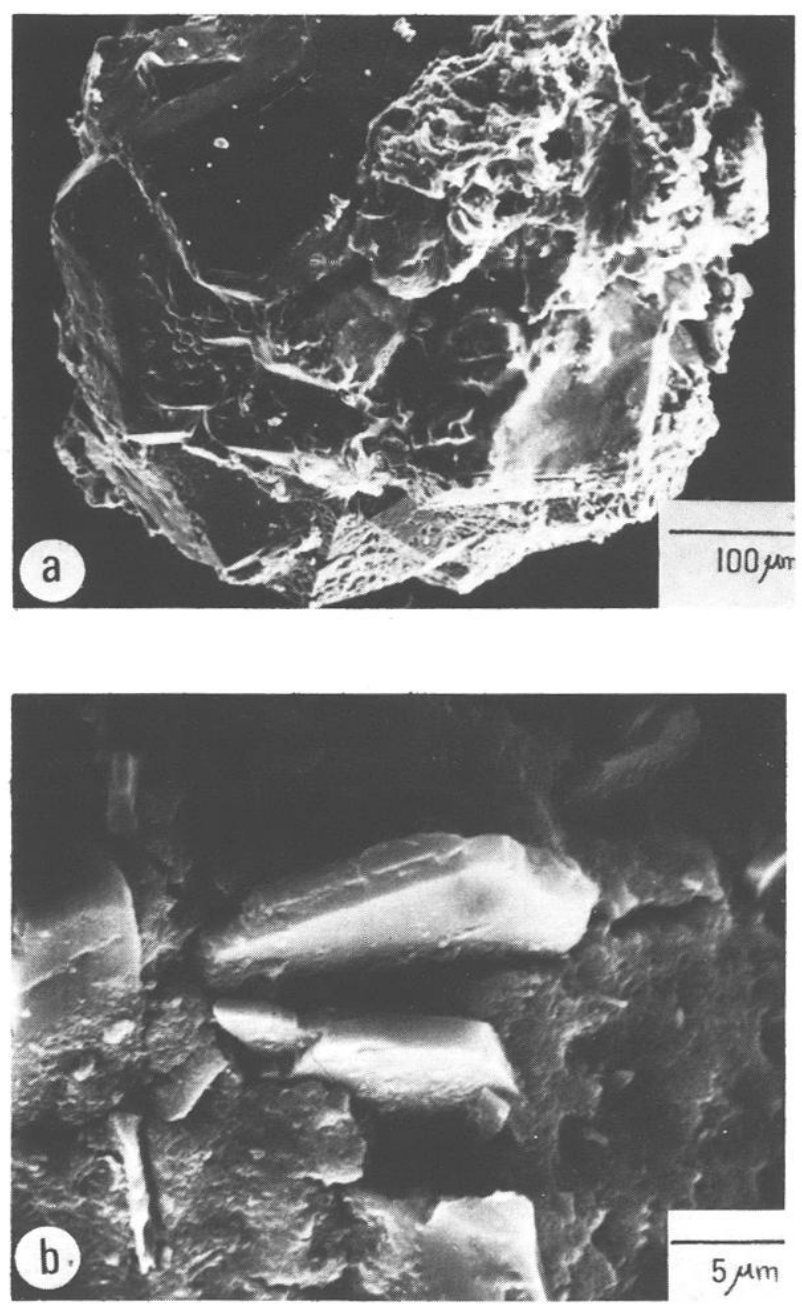

FIG. 4.

a) Agrégat de cristaux d'analcime (éch. : $53-4 / 80-82 \mathrm{~cm}$, unité V).

b) Plaquettes de clinoptilolite dans un ciment argileux (éch. : 28-1/65-67 cm, unité II).

On note que la distinction clinoptilolite-heulandite est difficile à effectuer par diffraction des rayons $\mathrm{X}$. Selon Mumpton (1960), la clinoptilolite est le terme le plus siliceux de la famille des heulandites et, généralement, elle contient plus de cations monovalents que divalents. Pour Mason et Sand (1960), la différence entre clinoptilolite et heulandite ne dépend pas de la teneur en Si mais de la nature des cations. La clinoptilolite a été défınie par ces auteurs comme une zéolite à structure d'heulandite dans laquelle $(\mathrm{Na}+\mathrm{K})>\mathrm{Ca}$. En nous référant à la classification de Mumpton, nous appelons clinoptilolites les zéolites tabulaires à fort rapport $\mathrm{Si} / \mathrm{Al}$. Lorsque la teneur en calcium est supérieure à celle du sodium, nous précisons " clinoptilolites calciques $»$.

b) Unités moyennes. (Sommet de IV et III.) L'analcime a disparu. On trouve une zéolite fibreuse, la mordénite, associée à une clinoptilolite calcique. Cette dernière est prédominante; elle forme de 90 à $95 \%$ de la paragenèse et de 7 à $8 \%$ du sédiment total. Ses caractères morphologiques sont les mêmes que plus 

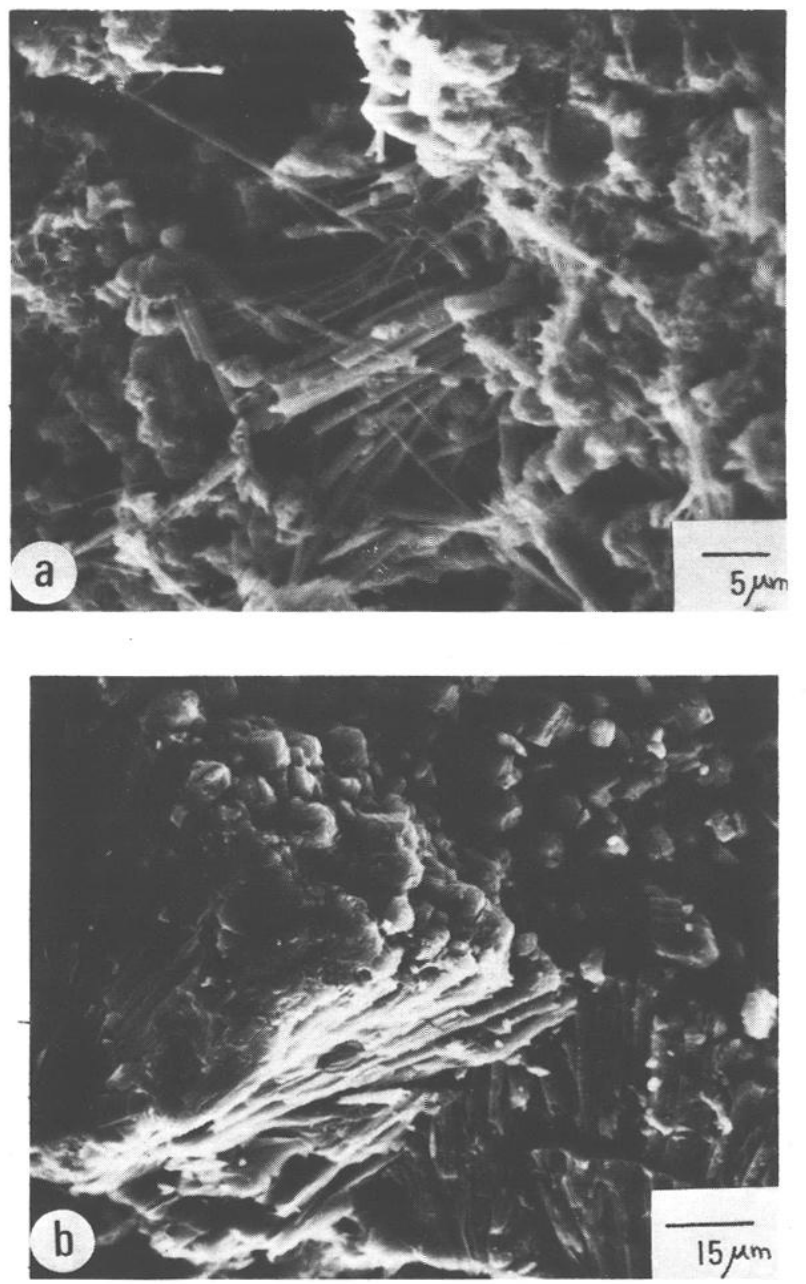

FIG. 5.

a) Fibres de mordénite (éch. : 42-1/105-107 cm, unité II). b) Fibres de phillipsite (éch. 28-1/65-67 cm, unité II).

bas. La mordénite (Figure $5 a$ ) se remarque par sa texture en gerbes de quelques $\mu \mathrm{m}$ de long. Elle possède un rapport $\mathrm{Si} / \mathrm{Al}$ élevé (entre 4,5 et 5,20 ) et elle est fortement sodique.

c) Unités supérieures. (II et I.) La mordénite n'existe plus et la phillipsite ( 7 à $8 \%$ du sédiment total) accompagne une clinoptilolite calcique identique à celle des unités sous-jacentes (Figure $4 b$ ). La phillipsite montre une texture soit massive dans les fissures du sédiment, soit prismatiquc dans des géodes (Figure 5 b). Son rapport $\mathrm{Si} / \mathrm{Al}$ est compris entre 1,90 et 3,0 et elle est très sodique $(\mathrm{Na}>\mathrm{K}$, traces de $\mathrm{Ca})$.

\section{2) Les smectites (Figures 2 et 6; tableau II).}

Elles constituent 90 à $95 \%$ de la fraction argileuse et $20 \%$ en moyenne du sédiment global. Comme il existe toujours dans nos échantillons un mélange de smectites dioctaédriques et de smectites trioctaédriques, nous avons estimé un paramètre $b$ moyen, donné par les rapports des principaux pics de la bande 060 , à l'échelle de l'échantillon d'abord, de l'unité ensuite. Ce paramètre augmente avec l'enfouissement; il indique que les smectites des unités basales sont riches en

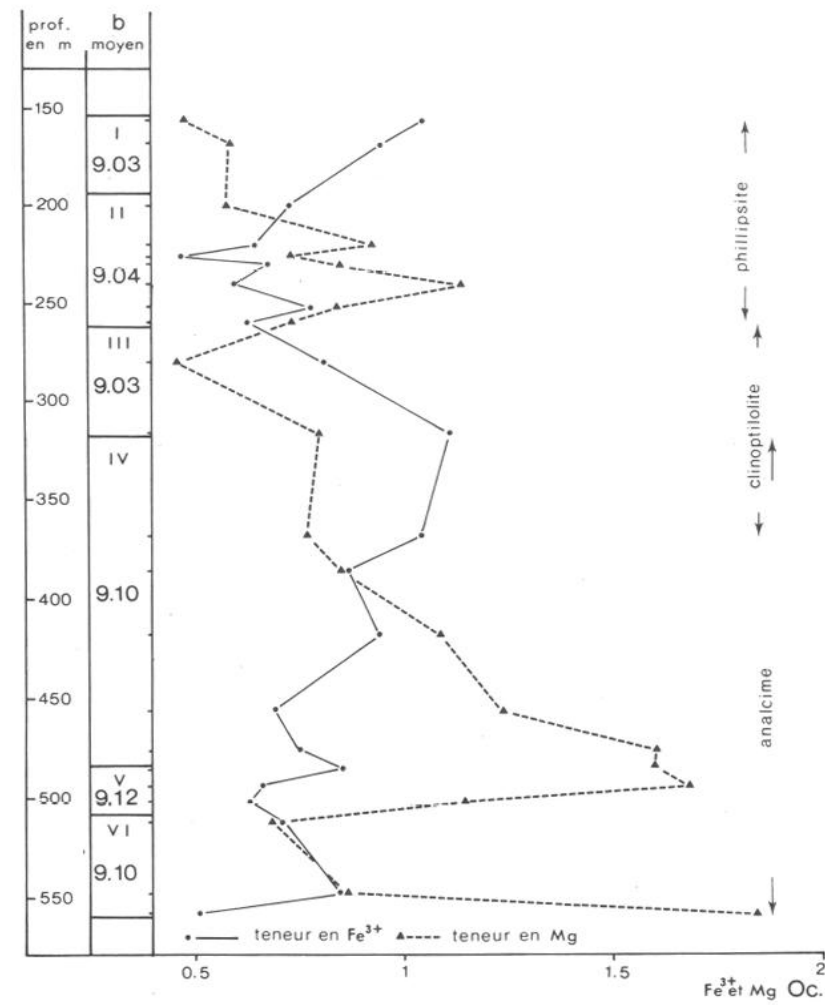

FIG. 6. - Teneur en $\mathrm{Fe}^{3+}$ et $\mathrm{Mg}^{2+}$, en fonction de la profondeur, dans la fraction argileuse, $<2 \mu \mathrm{m}$, composée de smectites (analyses spectrométriques).

- Abscisse : nombre de cations $\mathrm{Fe}^{3+}$ et $\mathrm{Mg}^{2+}$ en position octaédrique.

- Ordonnée : paramètre $b$ moyen, calculé à partir de la bande (06-33).

\begin{tabular}{|c|c|c|c|c|c|c|c|}
\hline Echantillons : & $\mathrm{SiO}_{2}$ & $\mathrm{Al}_{2} \mathrm{O}_{3}$ & : $\mathrm{MgO}$ & $\mathrm{Fe}_{2} \mathrm{O}_{3}$ & $\mathrm{Na}_{2} \mathrm{O}$ & $\mathrm{K}_{2} \mathrm{O}:$ & $\mathrm{CaO}$ \\
\hline $\begin{array}{r}\text { Saponite } \\
n^{2} 53-4\end{array}$ & 49.77 & 10.24 & 19.85 & 15.56 & 1.97 & 0.11 & 2.49 \\
\hline$\underset{\mathrm{n}}{\operatorname{Saponite}} \mathrm{Fe}$ : & 61.76 & 9.09 & 11.07 & 16.22 & - & 1.85 & - \\
\hline $\begin{array}{l}\text { Beide } 1 \text { jite } \mathrm{Fe} \\
\mathrm{n}^{\circ} 17-2\end{array}$ & 61.54 & 12.38 & 4.63 & 17.99 & : & 1.93 & 1.52 \\
\hline$\underset{\mathrm{n}^{\circ} 30-1}{\text { Céladonite }}$ & 59.25 & 2.92 & 9.46 & 26.37 & - & 4.32 & 0.32 \\
\hline
\end{tabular}

\begin{tabular}{|c|c|}
\hline Echantillons & Formules structurales \\
\hline $\begin{array}{l}\text { Saponite } \\
n^{\circ} 53-4\end{array}$ & 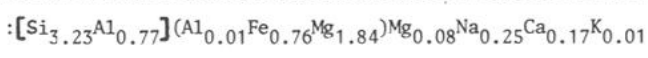 \\
\hline $\begin{array}{l}\text { Saponite } \mathrm{Fe} \\
\mathrm{n}^{\circ}{ }_{45-2}\end{array}$ & $:\left[\mathrm{Si}_{3.87}{ }^{\mathrm{Al}} 0.13\right]\left(\mathrm{Al}_{0.54} \mathrm{Fe}_{0.76} \mathrm{Mg}_{1.03}\right) \mathrm{K}_{0.15}$ \\
\hline $\begin{array}{l}\text { Beidellite } \mathrm{Fe} \\
\mathrm{n}^{\circ} 17-2\end{array}$ & 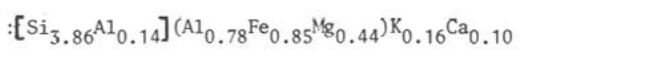 \\
\hline $\begin{array}{l}\text { Celadonite } \\
n^{\circ} 30-1\end{array}$ & 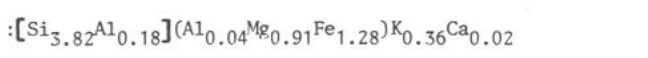 \\
\hline
\end{tabular}

TABlEAU II. - Exemples de composition chimique de smectites et de céladonite (analyses spectrométriques), et formules structurales.

magnésium $(b$ moyen $\simeq 9,10)$ et sont représentées essentiellement par des saponites riches en fer tandis que celles des unités supérieures correspondent surtout à des beidellites ferrifères ( $b$ moyen $\simeq 9,03$ ). L'analyse de poudres $\leqslant 2 \mu \mathrm{m}$ ou d'agrégats de smectites sur des fragments d'échantillons (Figures $7 a, 7 b$ ) confirme 

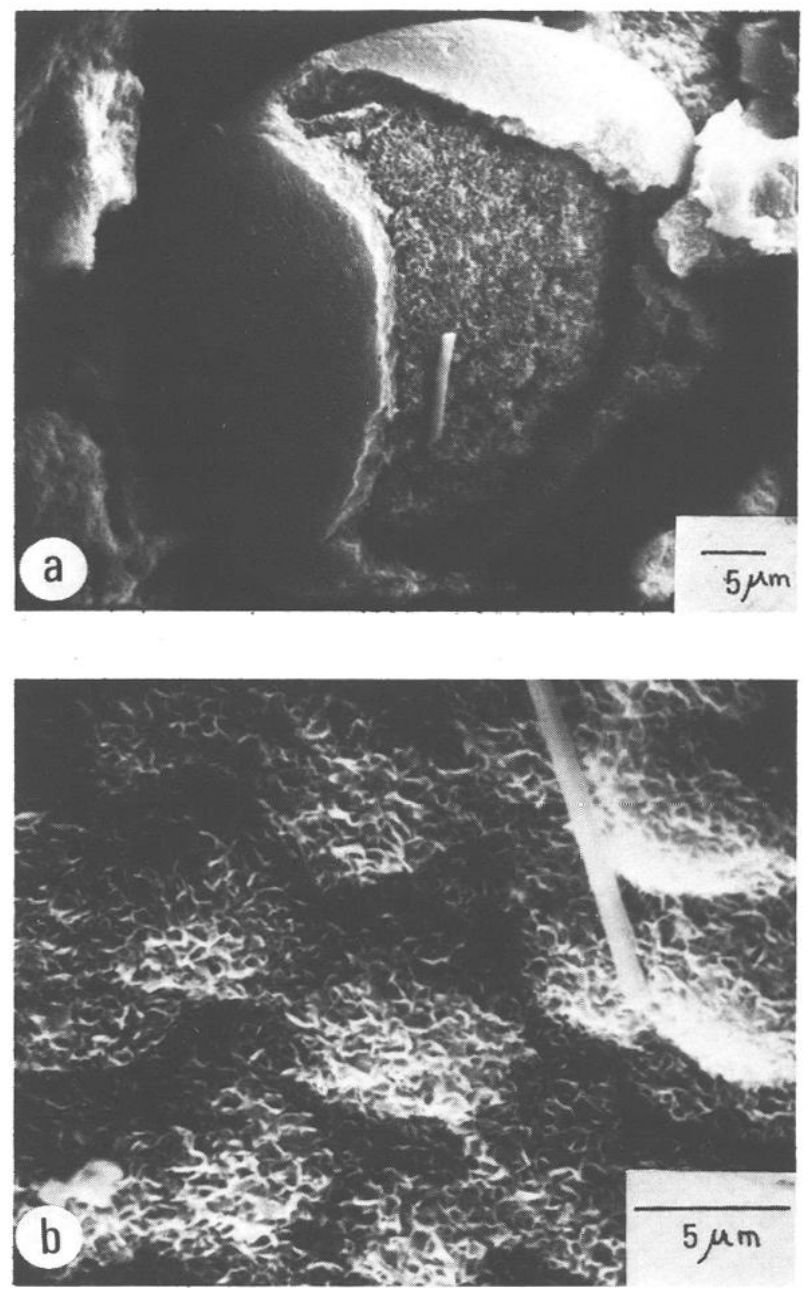

FIG. 7.

a) Boule de smectite (éch. : 54-4/80-82 cm, unité V). té V).

b) Smectite en " nid d'abeille " (éch. : $54-4 / 80-82 \mathrm{~cm}$, uni-

cette tendance; elle montre en outre, que la teneur en $\mathrm{Al}_{2} \mathrm{O}_{3}$ et $\mathrm{SiO}_{2}$ est constante quelle que soit l'unité.

\section{B) Les minéraux associés (Figure 2).}

\section{1) Les minéraux argileux annexes.}

Ils forment quelques $\%$ de la fraction argileuse totale et sont constitués par de la chlorite, des minéraux interstratifiés, surtout présents dans les unités inférieures, et de l'illite, régulièrement répartie dans toute la série.

Nous appelons «illite » tout minéral diffractant à $10 \AA$. Dans quelques échantillons (Tableau II), nous pouvons préciser que cette «illite », par sa composition chimique, la faible valeur de la substitution tétraédrique $(0,0$ à 0,3$)$ et son rapport $\mathrm{Fe} / \mathrm{Mg}$ voisin de 1,5 est très proche de céladonites décrites par Wise et Eugster (1964); on la trouve généralement dans le sédiment en remplissage de fissures. Les minéraux interstratifiés, étudiés sur les agrégats orientés, ont été identifiés comme étant de type "illite-smectite». D'après la méthode de Reynolds et Hower (1969), la quantité de feuillets d'illite dans ces interstratifiés varie entre 20 et $40 \%$.

\section{2) La calcite.}

Sa teneur dans toute la série est élevée et comprise entre 20 et $50 \%$ du sédimental total. Dans les unités supérieures elle est surtout d'origine biogénique (débris de macrofossiles). Dans les unités inférieures, elle cimente en gros cristaux les fragments vitreux.

\section{3) Les feldspaths.}

Ils sont abondants dans toutes les unités. Les examens au M.E.B. et les analyses montrent que les plagioclases (andésine) détritiques domirınt très largement les feldspaths potassiques authigènes.

\section{4) La silice.}

Elle est moins importante que la calcite et les feldspaths; mais elle peut atteindre (sous forme de quartz), $20 \%$ du sédiment total dans les unités inférieures.

Dans l'unité I, elle existe en lépisphères d'opale CT, mais la plupart du temps, elle se présente en grains sans forme distincte. L'unité III renferme quelques Diatomées.

\section{INTERPRÉTATION}

De bas en haut de la série des hyaloclastites, nous observons donc trois assemblages minéralogiques distincts :

- dans les unités basales, analcime et clinoptilolite sont associées à une smectite de type saponite;

- dans les unités moyennes, clinoptilolite et mordenite accompagnent une saponite de plus en plus ferrifère ;

- dans les unités supérieures, phillipsite et clinoptilolite coexistent avec une smectite de type beidellite ferrifère.

Plusieurs facteurs, non exclusifs, peuvent expliquer cette succession de paragenèses.

1) une modification du milieu de sédimentation au cours du dépôt,

2) une composition chimique des hyaloclastites variant en fonction du temps,

3) un changement dans les paramètres physicochimiques de l'altération.

D'après les données paléontologiques fournies sur l'environnement des hyaloclastites et le mécanisme de formation invoqué pour elles (voir supra), la première hypothèse ne semble pas devoir être retenue ici. Les deux autres possibilités sont plus à envisager.

\section{A) Composition chimique des hyaloclastites.}

Les basaltes présents dans le site 253 sont de 2 types; le basalte sous-jacent aux hyaloclastites (fortement altéré), est à olivine normative, alors que le basalte interstratifié dans l'unité II, relativement frais, est à quartz normatif. 
Cette différence dans la nature des 2 basaltes pourrait traduire une modification du chimisme des hyaloclastites au cours du temps. Un bilan géochimique effectué à aluminium constant permet de constater :

- une augmentation des alcalins ( $\mathrm{K}, \mathrm{Na}$ ) des hyaloclastites par rapport aux basaltes. Ceci, reflété dans la présence des zéolites sodiques (analcime, mordenite) et sodi-potassiques (phillipsite) est, vraisemblablement, en rapport avec les solutions altérantes;

- une augmentation du fer total de l'unité VI vers l'unité I, et une légère diminution du magnésium de l'unité I vers l'unité VI. Cela apparaît surtout au niveau des smectites formées de saponite dans les unités basales et de beidellite $\mathrm{Fe}$ dans les unités supérieures. Dans ce cas, et à la différence des alcalins, on ne peut exclure l'hypothèse que le changement du rapport $\mathrm{Fe} / \mathrm{Mg}$ dans la série ne tienne aussi en partie au précurseur. Toutefois, la distribution des terres rares dans les hyaloclastites reste tout au long de la séquence très homogène et semblable aux basaltes (Fleet et al., 1976), ce qui rend peu plausible une variation importante de la composition chimique des sédiments au cours de l'Éocène.

\section{B) Paramètres physico-chimiques de l'altération.}

\section{1) Nature des solutions.}

Des dosages effectués dans les eaux interstitielles tout au long du site 253 (Davies et al., 1974; McDuff, 1978) montrent que l'alcalinité des eaux reste faible, de l'ordre de $0,30 \mathrm{meq} / 1$, mais que la salinité augmente régulièrement de l'unité I vers l'unité VI $(36,6 \mathrm{~g} / 1$ à $42,10 \mathrm{~g} / \mathrm{l})$. On note également un net accroissement de la teneur en calcium (10 à $130 \mathrm{mM})$ avec la profondeur mais une diminution de celle en magnésium (55 à $5 \mathrm{mM}$ ).

Pour de nombreux auteurs, la teneur en ions $\mathrm{Na}$ et $\mathrm{K}$ des solutions conditionne la nature des zéolites. Des travaux expérimentaux (Keene et al., 1976) ont prouvé qu'avec un rapport $\mathrm{Na} / \mathrm{K}<0,5$, aucune zéolite ne pouvait se former. Pour un rapport supérieur et croissant, la phillipsite cristallise en premier, suivie de l'analcime. Höller et Wirsching (1978) n'ont obtenu que de l'analcime en système fermé; avec épuisement ou renouvellement des solutions altérantes, ils ont observé une succession de zéolites potassiques, puis sodiques. L'augmentation du calcium est causée par des phénomènes de dissolution ou de recristallisation de $\mathrm{CaCO}_{3}$, mais aussi par l'altćration de phases minérales riches en calcium, telles que les plagioclases (Anderson et al., 1976). Quant au magnésium, sa diminution est à relier à la cristallisation de minéraux argileux de type saponite (Perry et al., 1975).

\section{2) Rôle de la température.}

Nous pouvons essayer d'estimer les températures d'altération auxquelles ont été soumises les hyaloclastites d'après les domaines de stabilité de minéraux authigènes identiques à ceux que nous avons observés dans le site 253 et trouvés dans des conditions de gisement proches de ce dernier. Étant donné la nature et la faible épaisseur de la série, nous nous référons donc surtout aux températures des hydrothermes de faible pression, mesurées dans du matériel d'origine volcanique.

Dans les zones géothermiques de l'Islande (150 à $200^{\circ} \mathrm{C}$, à $1 \mathrm{~km}$ de profondeur), les smectites magnésiennes sont stables jusqu'à $200^{\circ} \mathrm{C}$. Au-delà, dans un domaine compris entre 200 et $280^{\circ} \mathrm{C}$, apparaissent des minéraux interstratifiés du type chlorite-smectite (Kristmannsdöttir, 1976). Mais en régime de diagenèse d'enfouissement où intervient plus directement la pression, sous 3000 à $5000 \mathrm{~m}$ d'épaisseur de sédiment, la corrensite, associée à l'analcime, est stable à des températures plus basses ( 95 à $150^{\circ} \mathrm{C}$ ), (Kübler, 1973). Au cours des processus de palagonitisation des basaltes, le stade initial de l'halmyrolyse est caractérisé, selon Honnorez (1978), par la paragenèse d'une phillipsite sodique $(\mathrm{Na}>\mathrm{K})$ ou de l'analcime et d'une smectite magnésienne, alors que le stade final est marqué par une smectite ferrifère et une phillipsite potassique ( $\mathrm{K}>\mathrm{Na}$ ). Melson et Thompson (1973) signalent également l'association d'une saponite ferrifère à l'analcime dans les produits de l'altération "hydrothermale » in situ des basaltes médio-atlantiques. Nous avons noté un peu de chlorite et de minéraux interstratifiés illitesmectite dans les unités basales des hyaloclastites, mais en teneur si faible que l'on ne peut exclure leur origine détritique. En conséquence, nous retenons une température maximale de $200^{\circ} \mathrm{C}$ à la base du site, avec un gradient que l'on peut préciser avec les zéolites.

Dans les mêmes zones géothermiques d'Islande, la phillipsite est stable jusqu'à approximativement $80^{\circ} \mathrm{C}$, la mordenite entre 80 et $230^{\circ} \mathrm{C}$ et l'analcime entre 70 et $300^{\circ} \mathrm{C}$ (Kristmannsdöttir et Tómasson, 1978). Mais, pour Iijima (1978), une transformation clinoptilolite $\mathrm{Na}$ ou mordenite-analcime s'opère de 84 à $91^{\circ} \mathrm{C}$, à une profondeur de 1,7 à $3,5 \mathrm{~km}$, dans des tuffs acides; la profondeur d'enfouissement a moins d'importance que la concentration en $\mathrm{Na}$ des eaux interstitielles, la température de l'équilibre étant abaissée par l'augmentation de cette concentration. Cet auteur note par ailleurs, vers $120-125^{\circ} \mathrm{C}$ et entre 2,5 et $4,5 \mathrm{~km}$ de profondeur, une réaction de l'analcime et du quartz avec formation d'albite pour des concentrations en $\mathrm{Na}$ des eaux interstitielles $\left(1 \times 10^{4} \mathrm{ppm}\right)$, tout à fait comparables à celles du site 253. Avec des concentrations plus faibles la température de l'équilibre est accrue. Nous n'avons pas trouvé d'albite dans le site 253. En défınitive, la température maximale atteinte peut être abaissée de $200^{\circ} \mathrm{C}$ (smectites) à $120-125^{\circ} \mathrm{C}$ et le domaine de formation de l'analcime apparaît alors compris entre cette température et $80-90^{\circ} \mathrm{C}$.

\section{CONCLUSION}

Les paragenèses observées dans le site 253 et leur zonation s'expliquent mieux par un gradient de température et la nature des eaux interstitielles que par un changement au cours du temps de la composition chimique des hyaloclastites.

En milieu marin, une distribution zonale de minéraux authigènes peut se produire dans des environne- 
ments géologiques distincts : diagenèse d'enfouissement, métamorphisme de contact, à la suite de l'intrusion de sills basaltiques par exemple, ou hydrothermalisme s.l. (Hay, 1978 ; Ijijima, 1978). La première possibilité n'est pas à envisager ici étant donné la faible épaisseur de la série; la deuxième pourrait l'être si l'on avait trouvé au contact des laves un début de "cuisson » ou tout au moins d'induration des hyaloclastites; la troisième est la plus plausible : dans une situation comparable à l'Islande, nous pensons que les sédiments volcanogéniques de la ride du $90^{\circ}$ Est ont été soumis à l'Éocène à une altération hydrothermale dans une aire géothermique de relative haute température (approximativement $200^{\circ} \mathrm{C} / \mathrm{km}$ ). Cette altération dans l'eau de mer a dû être rapide. En effet, la distribution des terres rares dans les smectites (Desprairies et BonnotCourtois, 1980), dans la céladonite et les zéolites du site 253 (travail en cours) ne montre que de faibles anomalies positives ou négatives en cerium ou même leur absence. Par ailleurs, les faibles teneurs ( $<20$ ppm) observées dans la céladonite et les zéolites sont à rapprocher de celles trouvées dans les dépôts hydrothermaux s.s. actuels (Bonnot-Courtois, 1981, sous presse).

\section{REMERCIEMENTS}

Ce travail a bénéficié de l'aide financière du Centre National pour l'Exploitation des Océans dans le cadre du contrat 79/5970. Les auteurs expriment leur gratitude au Professeur Miriam Kastner (Scripps Institution of Oceanography) pour ses conseils et remercient le Deep Sea Drilling Project d'avoir mis à leur disposition des échantillons du leg 26.

Reçu le 4 juin 1980. Accepté le 8 décembre 1980.

\section{RÉFÉRENCES}

Anderson, T. F., Donnely, T. W., Drever, J. I., EslinGer, E., Gieskes, J. M., Kastner, M., LaWrence, J. R. and Perry, E. A. (1976). - Geochemistry and diagenesis of deep-sea sediments from leg 35 of the Deep Sea Drilling Project. Nature, vol. 261, 473-476.

Bonnot-Courtois, C. (1981). - Distribution des terres rares dans les dépôts hydrothermaux de la zone $\mathrm{Fa}$ mous et des Galapagos. Comparaison avec les sédiments métallifères. Marine Géol. $v .39$ (sous presse).

Davies, T. A., Luyendyl, B. P., Rodolfo, K. S., Kempe, D. R. C., MCKelvey, B. C., Leidy, R. D., Horuath, G. J., Hyndman, R. D., Thierstein, H. R., Herb, R. C., Boltovshoy, E. and Doyi.F, P. (1974). - Initial reports of the Deep Sea Drilling Project, 26. U.S. Govt. Printing Office, Washington, D.C., 1129 pp.

Desprairies, A. et Bonnot-Courtois, C. (1980). - Relation entre la composition des smectites d'altération sousmarine et leur cortège de terres rares. Earth. Plan. Sci. Lett., 48, 124-130.

Fleet, A. J., Henderson, P. and Kempe, D. R. C. (1976). Rare earth element and related chemistry of some drilled southern Indian Ocean basalts and volcanogenic sediments. J. Geophys. Res., 81, 4257-4268.

HAY, R. L. (1978). - Geologic occurrence of zeolites. In : Natural zeolites. Occurrence, properties, use. Sand, L.B. and Mumpton, F.A. ed. Pergamon Press., 135-143.

Höller. H. and WIRSChING, U. (1978). - Experiments on the formation of zeolites by hydrothermal alteration of volcanic glasses. In : Natural zeolites. Occurrence, properties, use. Sand, L. B. and Mumpton, F. A. ed. Pergamon Press., 329-336.

Honnorez, J. (1978). - Generation of phillipsite by palagonitization of basaltic glass in sea water and origin of $\mathrm{K}$ rich deep sea sediments. In : Natural zeolitesOccurrence, properties, use. Sand, L. B. and Mumpton, F. A. ed. Pergamon Press, 245-258.

IIJimA, A. (1978). - Geological occurences of zeolites in marine environments. In : Natural zeolites. Occurrence, properties, use. Sand, L. B. and Mumpton, F. A. ed. Pergamon Press., 175-198.

Keene, J. B., Clague, D. A. and Nishimori, R. K. (1976). Experimental hydrothermal alteration of tholeitic basalt : resultant mineralogy and textures. J. of. Sed. Petrol., vol. 46, $\mathrm{n}^{\circ} 3,647-653$.
KRISTMANNSDÖTTIR, H. (1976). - Types of clay minerals in hydrothermally altered basaltic rocks, Reykjanes, Iceland. Jökull, 26, 30-39.

KRISTMANNSDörtrir, H. and Tómasson, J. (1978). - Zeolite zones in geothermal areas in Iceland. In : Natural zeolites. Occurence, properties, use. Sand, L. B. and Mumpton, F. A. ed. Pergamon Press., 277-284.

KüBLER, B. (1973). - La corrensite, indicateur possible de milieux de sédimentation et du degré de transformation d'un sédiment. Bull. Centre Rech. Pau, S.N.P.A., 7, 2, 543-556.

McDuff, R. E. (1978). - Conservative Behavior of calcium and magnesium in the interstitial waters of marine sediments : identification and interpretation. $\mathrm{Ph}$. D. Thesis. University of California San Diego.

MCKelvey, B. C. and Fleet, A. J. (1974). - Eocene basaltic pyroclastics at site 253 , Ninetyeast Ridge. In : Davies, T. A., Luyendyk, B. P. et al. Initial reports of the Deep Sea Drilling Project, 26 U.S. Govt Printing Office, Washington, D.C., 553-565.

MASON, B. and SAND, L. B. (1960). - Clinoptilolite from Patagonia The relationship between clinoptilolite and heulandite. Amer. Mineral., vol. 45, 341-350.

Melson, W. and Thompson, G. (1973). - Glassy abyssal basalts atlantic sea floor near St Paul'rocks : petrography and composition of secondary clay minerals. $G$. Soc. of Am. Bull., Vol. 84, 703-716.

Mumtron, F. A. (1960). - Clinoptilolite redefined. Amer. Mineral., vol. 45, 351-369.

Perry, E. A., Gieskes, J. M. and Lawerence, J. R. (1976). $\mathrm{Mg}, \mathrm{Ca}$ and $\mathrm{O}^{18} / \mathrm{O}^{16}$ exchange in the sediment-pore water system, Hole 149, D.S.D.P. Geoch. et Cosmoch. Acta, vol. 40, 413-423.

REYNOLdS, R. C. and Hower, J. (1969). - The nature of interlayering in mixed-layer illite-montmorillonite. Clays and Clay Miner., 1970, vol. 18, 25-36.

SAHA, P. (1959). - Geochemical and X-ray investigation of natural and synthetic analcites. Amer. Mineral., Vol. 44 , 330-313.

TAziefF, H. (1952). - About deep sea volcanism. Geol. Rundsch., 61 (2), 470-480.

Wise, W. S. and Eligter, H. P. (1964). - Celadonite : synthesis, thermal stability and occurrence. Amer. Mineral., Vol. 49, 1031-1083. 